\title{
Knob Device
}

National Cancer Institute

\section{Source}

National Cancer Institute. Knob Device. NCI Thesaurus. Code C50022.

A handle, usually attached to a shaft allowing manual rotation of the shaft. 